\title{
Designing number of Kanban in glove box assembly
}

\author{
Chonthicha Limpayanak ${ }^{1, *}$ and Oran Kittithreerapronchai ${ }^{1, *}$ \\ ${ }^{1}$ Industrial Engineering Department, Faculty of Engineering, Chulalongkorn University, Bangkok, Thailand
}

\begin{abstract}
For many decades, Just-In-Time manufacturing and lean manufacturing have adopted kanban system as a mean to control inventory and production. A number of tagged kanban cards represents inventory of the system, whereas untagged kanban cards equate to production orders. The ideal conditions are a consistent product mixed with a small production lot size and minimum setup time. Such conditions are difficult to obtain even in many automotive assembly lines similar to a case study. The case study assembly line is a glove box assembly production line that assembles two plastic parts and wields them together with a vibration machine after positioning in a mold designed for different products. Because of storage equipment, each kanban card represents 60 identical glove boxes. Therefore, a production engineer re-schedules the production planning to minimize the numbers of changeover without any effect on downstream. This ad hoc production scheduling leads to urgent production orders and interruption of lot size. As a result, the factory embeds historical data into a computer simulation that accounts for current molds, receiving time, and numbers of pending kanban cards, as well as number of storage equipment. The results of this simulation reveals that scheduling criteria and suggest a suitable lot size if the factory decides to change a storage equipment.
\end{abstract}

\section{Introduction}

Pioneered by Toyota, Just-In-Time (JIT) and lean manufacturing systems have become synonymous with a pull system and adopted kanban system as a mean to control inventory and production. Many researchers and practitioners view kanban system as a controlling mechanism that monitors a constant work-in-process or base stock system [1].

Particularly, a number of tagged kanban cards represents inventory of the system, whereas untagged kanban cards equate to production orders. In the ideal conditions, each individual part, including nuts and bolts, should have their own kanban cards and a consistent product mixed with a small production lot size and a minimal setup time. Nevertheless, such conditions are difficult, if not impossible, to obtain even in many automotive assembly lines because the trade-off between the efficiency in batch production and/or batch transportation and the responsiveness in production changeover. Hence, the goal of this article is to address and analyse the trade-off using the production of a case study assembly line as an example.

Similar to many manufacturing plant, the case study company embraces a pull system with kanban system to manufacture plastic components used in the automotive industry. The case study assembly line is a glove box assembly, as shown in Figure 1.

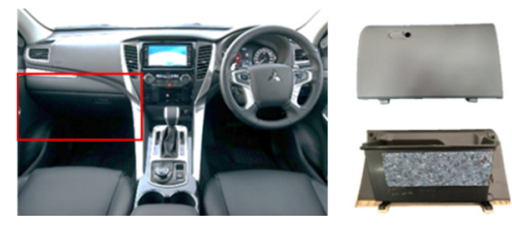

Fig. 1. Example of glove boxes manufactured in a case study company.

At the time of writing, the company manufactures eleven models that consist of two different molds for different passing lane, particularly left-hand-side (LHS) and right-hand-side (RHS) driving. Because of the capacity of a mobile rack used as storage and transportation equipment, each kanban cards equivalents to 60 glove boxes with an identical model. Therefore, a production engineer must re-schedule the production planning to address that the trade-off between minimizing the numbers of changeover and possible product shortage downstream. Before evaluating the production scheduling criteria and design scheduling and suggesting a suitable lot size if the company decides to change a mobile rack, it is important review relevant literature.

\section{Literature Review}

Sequencing the glove box production planning in an automotive assembly line has related to two interdependent streams of research JIT Manufacturing System and Number of kanban. 


\subsection{JIT Manufacturing System}

As a control production and inventory control mechanism in JIT manufacturing system, a kanban card is used to transmit inventory and production by tagged or untagged with items, respectively. Managing a number of kanban cards is one of key successes of JIT manufacturing system as it must ensure production synchronization between different department while maintains minimum inventory. In addition to analyze the formulation used Toyota to determine kanban cards, Monden [2] applied the Little's law by determining an appropriated safety factor, demand rate, lead time, and container capacity. Since then literature has described and proposed both optimal one and/or heuristic processes for different situation. For example, Philipoom et al [3] proposed an Integer Linear Programming to determine an optimal number of kanban cards for multistage and multi-product assembly line. Few researchers have addressed operational issues as majority of literature assumed that the variation of processing time is small as well as ignored setup time and sequencing of kanban cards. One empirical study [5] found that four factors affect number of kanban cards are throughput rate, variation in processing time, utilization of machine, and continuity of processing time. An interesting reader can consult [6] for the design of kanban card and operational issues.

Based on these literature, JIT System in the glove box assembly line can be classified as a single kanban card part-type and queue-size blocking mechanism with batch production system similar to Tayfus [7] that addressed multiple production type. Rather assuming Poisson distribution, this article explores empirical distribution of parts. Having reviewed and established the connection with the related literature, the remaining sections are organized as follows. Section 3 highlights and analyzes the problem in the glove box assembly line. After understanding nature of the assembly line, a discrete event time simulation is introduced in Section 4 whereas the result is reported and discussed in Section 5.

\section{Case Study Company}

Located in the eastern of Thailand, this company is the first tier manufacturer that has produced automotive plastic parts. Relied on kanban system, a case study company has used four different kanban based on different party, as shown in Figure 2.

Depending on a design shape and size, the main manufacturing process is the plastic injection process. In general, the company divides its plastic products into different categories based on its functions and installation locations inside an automotive vehicle. Located underneath a vehicle dashboard, a glove box is viewed as an instrument panel. Designed to storage documents or small items, a typical globe box design consists of two plastic parts and wields them together at the glove box assembly line.

\subsection{Glove Box Assembly line}

\footnotetext{
Corresponding author: climpayanak@gmail.com
}

The glove box assembly line comprises of two operators and single vibration machine. The purpose of the assembly line is to prepare and assemble the plastic parts with the vibration machine after positioning them in a mold designed for different product models, as shown in Figure 3.

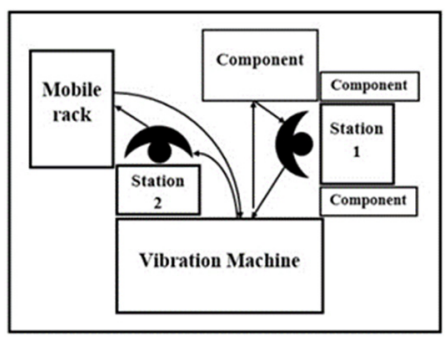

Fig. 2. Vibration machine used in the glove box assembly line.

Each operator has different roles. Before transferring the plastics parts to the vibration machine, the first operator manually assembles components onto glove box and positions the inner and outer parts of a glove box into their respective molds inside the vibration machine for welding. After the vibration welding completed, the second operator removes welded glove box and assemble additional components before packing in to a mobile rack for storage and transportation. In the change-over event, an addition operator is required to assist the assembly-line operators to remove and replace mold because of their size and weight. Without the changing over, this assembly line is a simple tendum line system with a single machine and two operators to load and unload a component to a machine. Hence, the trivial solution to maximize the utilization and throughput is to minimize the chance-over. Nevertheless, the mixed glove box requirement of downstream process as well as its JIT environment prohibits such solution. Therefore, it is interesting to analyze the change-over.

\subsection{Change-Over Analysis}

To improve the efficiency, the production engineer of the assembly line re-schedules the production planning to minimize the numbers of changeover, while observing three factors that indicate the downstream demands:

Duration time is the time in which a kanban card arrives can indicates consumption rate of downstream production. Assuming a single model assembly, the production engineer can estimate the consumption rate of recent arrival kanban. It is important to note that the interarrival time of a kanban card also help to validate this assumption.

The remaining kanban is the difference between available and total kanban cards. Because of a constant planning cycle, a number of kanban cards in each model every remains constant for ten days before revising in the next cycle. Therefore, a number of available kanban cards of each model with respected to its total reveals the inventory at store department and downstream production line.

Change-Over Sequence: In additional of an individual model, the relationship between current model 
and potential change-over model is also an important factor as some models may require complete changeover of mold, while the others may require only cleaning and additional processing time.

Based on these factors and personal experience, the engineer schedule production to balance between utilization and change-over downtime, as depicted in Figure 4.

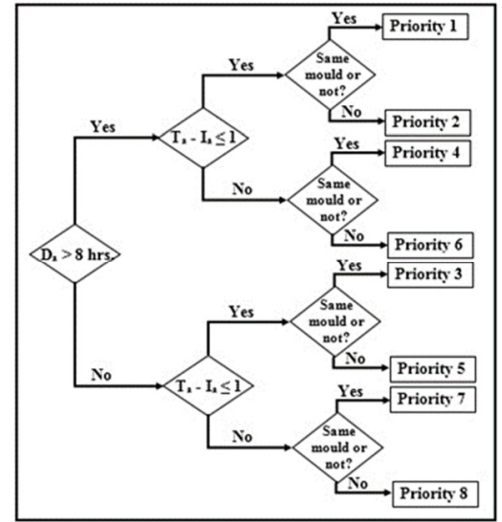

Fig. 4. Decision tree diagram of the current scheduling practice.

The practice of a simple decision tree diagram that ignores sequence of kanban cards has raised questions and concerns among department and led to the analysis of production sequence, as shown in Figure 5.

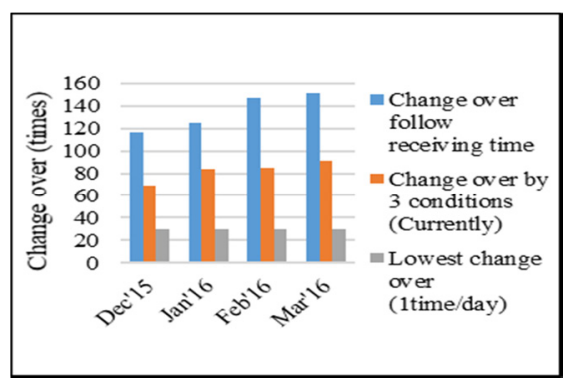

Fig. 5. Comparison of monthly change-over times between current practice and kanban sequence

Figure 5 reveals the advantage of the current practice. Particularly, scheduling by three factors could significantly reduce a number of change-over. This practice, however, lacks of theatrical ground as it relies heavily on the experience of an engineer without a useful insight on important factors. Furthermore, the past urgent request was usually done without any record. As a result, the case study company wish to analyze the most important factors including a capacity of mobile rack as well as to develop a suitable decision criteria. To answer these questions, a discrete event-time simulation model, as discussed in the next section.

\subsection{Processing Time}

To Before developing a simulation model, it is important to analyze the processing time of each operator as well as welding machine, as shown in Figure 6.

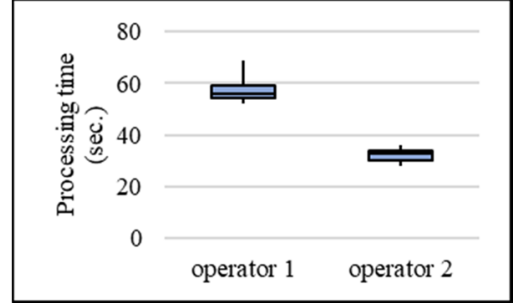

Fig. 6. Distribution of processing time of each operator

Figure 6 results from actual time and work of each operation denoted by his station. During the study, 30 samples of activity are randomly corrected and analyzed. The processing times of both operators are normally distributed with mean 57.0 and 35.0 seconds and variance 2.5 and 3.5 seconds, respectively. The processing time of vibration machine is 15 second regardless mold type and model.

\section{Glove Box Assembly Simulation}

The purpose of glove box assembly simulation is to determine effects and interaction of possible factors by simulating a manufacturing one production cycle or ten days with empirical schedule. Based on the decision diagram in Figure 4, three factors are introduced, partially.

The levels of each factor as well as the scenarios of experiments are based on current operation and extreme values of each factor as shown in Table 1.

Table 1. Scenarios and levels of factors in experiment

\begin{tabular}{|c|c|c|c|c|}
\hline Scenario & $\begin{array}{c}\mathbf{R} \\
\text { (piece) }\end{array}$ & $\begin{array}{c}\mathbf{D} \\
\text { (hours) }\end{array}$ & $\begin{array}{c}\text { K } \\
\text { (cards) }\end{array}$ & Note \\
\hline A0 & 60 & 8 & 1 & Current \\
\hline A1 & 30 & 8 & 1 & \\
\hline A2 & 90 & 8 & 1 & \\
\hline A3 & 60 & $\infty$ & $\infty$ & FIFO \\
\hline A4 & 60 & 4 & 1 & \\
\hline A5 & 60 & 6 & 1 & \\
\hline A6 & 60 & 10 & 1 & \\
\hline A7 & 60 & 16 & 1 & \\
\hline A8 & 60 & $\infty$ & 1 & \\
\hline A9 & 60 & 8 & 0 & \\
\hline A10 & 60 & 8 & 2 & \\
\hline A11 & 60 & 8 & $\infty$ & \\
\hline
\end{tabular}

Having defined factors and contemplated on values of each factor, each scenario is modeled as a discrete event time simulation model.

The operation flow of model begins with creating entities or kanbans with status associated score representing mold side, duration time, and available kanban. The model re-evaluates scores of every kanban time when a kanban is assigned to different operator, including created or exited the system. If the first operator is available, the simulation assigns the highest score kanban to the operator who assembles components into glove boxes and loads them into the vibration

\footnotetext{
* Corresponding author: climpayanak@gmail.com
} 
machine. The particular kanban is occupied until completed its batch size based on rack capacity.

\subsection{Result of Simulation}

The simulation is embedded in Rockwell Arena 16.5 with 100 replications. The model was verified by number of kanban comparing between a kanban that generated from simulation. The results of each scenario in terms of system performance and operator performance are shown in Table 2 and Table 3, respective.

With exception of a higher machine utilization, the performance of the current re-scheduling kanban (A0) dominates the performance of FIFO kanban (A3). This suggests a merit of re-scheduling kanban and leads to analysis of each factor.

Table 2. System preformance of each scenario

\begin{tabular}{|c|c|c|c|}
\hline Scenario & $\begin{array}{c}\text { number of } \\
\text { mold changed }\end{array}$ & $\begin{array}{c}\text { Glove box } \\
\text { completed } \\
\text { (pcs) }\end{array}$ & $\begin{array}{c}\text { Ratio completed } \\
\text { kanban }\end{array}$ \\
\hline A0 & $49.7 \pm 1.2$ & $8,583.5$ & 0.974 \\
\hline A1 & $73.3 \pm 1.6$ & $8,516.5$ & 0.974 \\
\hline A2 & $35.4 \pm 1.0$ & $8,611.8$ & 0.992 \\
\hline A3 & $68.3 \pm 1.5$ & $8,519.9$ & 0.993 \\
\hline A4 & $53.9 \pm 1.2$ & $8,545.9$ & 0.975 \\
\hline A5 & $51.4 \pm 1.2$ & $8,531.2$ & 0.974 \\
\hline A6 & $48.6 \pm 1.2$ & $8,551.2$ & 0.977 \\
\hline A7 & $46.5 \pm 1.2$ & $8,586.4$ & 0.975 \\
\hline A8 & $46.2 \pm 1.2$ & $8,581.9$ & 0.993 \\
\hline A9 & $49.7 \pm 1.2$ & $8,583.6$ & 0.974 \\
\hline A10 & $49.7 \pm 1.2$ & $8,583.6$ & 0.974 \\
\hline A11 & $49.7 \pm 1.2$ & $8,583.6$ & 0.974 \\
\hline
\end{tabular}

Table 3. Operator preformance of each scenario

\begin{tabular}{|c|c|c|c|}
\hline \multirow{2}{*}{ Scenario } & Machine & \multicolumn{2}{|c|}{ Cycle Time (hours) } \\
\cline { 3 - 4 } & Utilization & Avg & Max \\
\hline A0 & 0.22 & $2.24 \pm 0.21$ & 5.74 \\
\hline A1 & 0.25 & $2.79 \pm 0.21$ & 6.28 \\
\hline A2 & 0.20 & $1.90 \pm 0.20$ & 6.69 \\
\hline A3 & 0.24 & $2.69 \pm 0.27$ & 9.45 \\
\hline A4 & 0.22 & $2.27 \pm 0.22$ & 8.87 \\
\hline A5 & 0.22 & $2.16 \pm 0.19$ & 6.04 \\
\hline A6 & 0.21 & $2.22 \pm 0.22$ & 8.00 \\
\hline A7 & 0.21 & $2.15 \pm 0.18$ & 5.09 \\
\hline A8 & 0.21 & $2.11 \pm 0.17$ & 4.95 \\
\hline A9 & 0.21 & $2.24 \pm 0.21$ & 5.74 \\
\hline A10 & 0.21 & $2.24 \pm 0.21$ & 5.74 \\
\hline A11 & 0.21 & $2.24 \pm 0.21$ & 5.74 \\
\hline
\end{tabular}

The results of Scenarios A0 to A2 suggest that changing rack capacity greatly affects performance of system and operators. As the capacity increases, productivity increase as number of molds and average cycle time are reduced. Nevertheless, there is little impact to overall utilization as the utilization of vibration machine increase slightly. It is important to note that utilizations of the first operator and the second operator remain unchanged at 0.71 and 0.44 throughout the experiment.

Changing duration time required to process kanban leads to increase number of mold changed as it reduces probability of using the same mold especially LHS mold which has fewer models that RHS mold. Interestingly, the benefit of duration is marginal decreasing and other performance indicators remain unchanged.

Based on Scenarios A0, A9, A10, and A11, the simulation reveals that remaining kanban has little or no effect to any performance indicators.

\section{Summary}

The article addresses and analyzes the trade-off between minimizing the numbers of changeover and possible product shortage down-stream in a glove box assembly. Using the production. Based on current decision tree to re-schedule and another suggested factor, the operation and re-scheduling procedure were modeled as a discretetime event simulation. The result suggests that changing rack storage capacity would greatly affect performance, whereas increasing duration before process kanban could reduce mold change-over with little effect on other performance indicators. The result also reveals that counting remaining kanban in system has no benefit for re-scheduling.

\section{References}

1 Karmarkar U., Push, pull and hybrid control schemes, Tijdschrift voor Economie en Management 26, 345-363 (1991)

2 Monden Y., Toyota Production System, Norcross, Georgia Industrial Engineering and Management Press, 215-216 (1983)

3 Philipoom P., Rees L., Taylor III B. and Huang P., A mathematical programming approach for determining workcentre lotsizes in a just-in-time system with signal Kanbans, 1-15 (1990)

4 Philipoom P., Rees L., Taylor III B. and Huang P., A mathematical programming approach for determining workcentre lotsizes in a just-in-time system with signal Kanbans, 1-15 (1990)

5 Philipoom P., Rees L. and Taylor III B. W., Simultaneously Determining the Number of Kanbans, Container Sizes, and the Final-Assembly Sequence of Products in a Just-in-Time Shop, International Journal of Production Research, Vol.34, 51-69 (1996)

6 Akturk M. S. and Erhun F., An overview of design and operational issues of kanban systems, International Journal of Production Research, Vol.37, 3859-3881 (1999)

7 Tayfur A., and Goang A. S., Pull-type manufacturing systems with multiple product types, IIE Transactions journal, Vol.32, 115-124 (2000)

\footnotetext{
Corresponding author: climpayanak@gmail.com
} 